\title{
Impact of COVID-19 Lockdown on Sleep Quality in Students
}

\author{
Pragya Tiwari, Minal Trivedi, Apeksha Kesarwani, Ojaswi Gopale \\ Department of Biotechnology, B.K Birla College of Arts, Science and Commerce (Autonomous), Kalyan. \\ Maharashtra, India \\ *Corresponding Author Email Id: iampragyat@gmail.com
}

Article Info

Volume 8, Issue 5

Page Number : 265-269

Publication Issue :

September-October-2021

\section{Article History}

Accepted : 10 Oct 2021

Published: 20 Oct 2021

\section{ABSTRACT}

A periodic state of rest accompanied by varying degrees of unconsciousness and relative inactivity is referred as sleep; in another way is a state in which an individual lacks conscious awareness of environment surroundings. Quality sleep and getting enough of it at the right times is as essential for survival just as food and water. Without sleep our brain can't learn and create new memories, making it harder to concentrate and respond quickly. The Novel Coronavirus (also known as COVID-19) ushered the world into uncharted waters. In India, strict lockdown was imposed in three phases from March to June 2020 for the containment of the COVID-19 pandemic. At this juncture, we attempted to assess how derailment of social life due to imposed social isolation, leading to compromised sleep in the present scenario affects circadian driven sleep-wake pattern and other lifestyle related behaviour. A brief survey on affected sleep pattern of people during corona pandemic was conducted to understand the possible alterations in sleep-wake schedules and the daily routine related activities such as exposure time to digital media (i.e., $\mathrm{TV}$, laptop/computer/mobile, etc.) as a consequence of lockdown.

Keywords : Covid-19 Pandemic, Sleep, Coronavirus, Insomnia, Lockdown.

\section{INTRODUCTION}

Sleep, the natural, easily reversible periodic state of many living things that is marked by the absence of wakefulness and by the loss of consciousness of one's surroundings, is accompanied by a typical body posture (such as lying down with the eyes closed), the occurrence of dreaming, and changes in brain activity and physiological functioning, is made up of cycles of non-REM sleep and REM sleep, and is usually considered essential to the restoration and recovery of vital bodily and mental functions. It is an important part of the daily routine where about one-third of time is spent. Sleep (or atleast a physiological period of quiescence) is a highly conserved behaviour that occurs in animals ranging from fruit flies to humans. So a result, a substantial fraction of human's life is spent in this mysterious state. Sleep disruptions can be passing problem or sign of a more serious underlying medical condition. Sleep is vital to human 
health and serves critically in neurobehavioral, cognitive and safety- related performance, memory consolidation, nociception as well appetite regulation, immune and hormonal functions [1]. COVID-19 crisis brought the entire world to a standstill. Countries established various levels of lockdowns, economies have ground to a halt and many people are afraid for themselves and their loved ones.

On March 25th 2020, nationwide lockdown was adopted to prevent disease or contamination but it created negative impact on people's mental health, suddenly people were limited to small houses. Previous studies have postulated that this type of isolation creates a negative impact on the psychological aspect on those who have infected and their families, which may lead to sleep disturbances and post-traumatic stress [2]. Lockdown to prevent the community transmission of COVID-19 pandemic has confined the people at home and imposed social restrictions, which is expected to cause alterations in circadian driven sleep-wake schedule and its associated lifestyle behaviours. Discord with social and natural cues due to complete lockdown during COVID-19 pandemic leads to a state of social jetlag with delayed sleep-wake, meal timings and excessive digital media exposure among Indians, which has differential impact on males and females as well as across different age groups [3].

There was a significant increase of psychological distress and symptoms of mental illness in the general population. Previous studies found that acute infectious disease, like SARS, can cause anxiety, depression, stress, and post-traumatic stress disorder both in survivors and non-infected people. Previous studies showed a negative impact on mental health on people that face sudden events that could even culminate in similar post-traumatic stress disorders symptomatology. Traumatic events such as those caused by COVID-19 outbreak can produce psychological distress and anxiety symptoms which negatively impact sleep quality. For this reason, researchers focused on physical and mental health, and sleep during COVID-19 emergency [4,5]

Data from an ongoing longitudinal study of a large representative sample of households indicated that one month after the World Health Organization's declaration, also estimates that about 7.5 per cent Indians suffer from some mental disorder and predicts that by end of this year roughly 20 per cent of India will suffer from mental illnesses. According to the numbers, 56 million Indians suffer from depression and another 38 million Indians suffer from anxiety disorders [7].

Staying at home during lockdown exposes people to extended artificial light or digital screens due to excessive use of virtual platforms in terms of TV, laptop, mobile etc. However, increased artificial light exposure is known to have adverse effect on sleep health, reducing its quality and increasing the day time sleepiness. Alteration in melatonin hormone, the chronobiotic conveying information about night/dark and therefore sleep timing to the brain, is known to be maximally affected by the late night artificial light exposure. Besides, higher screen time is also correlated to reduced physical activity level that interferes with the sleep quality. In addition, meal timing is known to act as a non-photic cue that entrains the circadian rhythm [3] .

As in India, strict lockdown was imposed in three phases from March to June 2020 for the containment of the COVID-19 pandemic. Most people were confined to their homes. This confinement is stressful in itself as individuals are sharing the limited space for a prolonged period with few close contacts. In addition, they experience a lack of novel stimuli, disruptions of routine activity, increased parenting responsibilities, especially for women, and altered productivity expectations for those engaged in professional duties from home. In addition to the 
ever-present fear of contracting COVID-19 as it spreads across the country, uncertainty about jobs, economic situation, and the health and safety of loved ones. The pandemic has also been described as an information epidemic, as most people have constant access to news about negative consequences, much of it through electronic media and consequent increase in "screen time." In short, lockdown resulted in home confinement during prevailing anxiety and reduction of positive stimuli. Stress, in general, but not always, has an inverse relationship with sleep. The effect of stress on sleep quality, timing, and duration is influenced by sleep reactivity. Persons with high reactivity develop insomnia during stressful situations while those without do not. Thus, home-confinement resulting from lock-down increases the chances of disturbed sleep and insomnia through stress $[8,9]$. Insomnia has been defined as a term to describe the presence of polysomnographic presence of disturbed sleep. Thus, the presence of long sleep latency, frequent nocturnal awakenings or prolonged periods of wakefulness during the sleep period or even frequent transient arousals are taken as evidence of insomnia. Insomnia is a risk factor for Major depression, anxiety disorders, substance used disorders, suicidality, diabetes, hypertension. Thus, insomnia has been thought of both the symptom and as a sign. The diagnosis of insomnia and the severity of the sleep disturbance are related to overactivation of the hypothalamic-pituitary-adrenal (HPA) axis and the hypersecretion of cortisol.Recent evidence suggests that there may be some neuroendocrine and clinical similarities between insomnia and depression. Corticotropin-releasing factor (CRF) dysregulation has been implicated in the pathogenesis of psychiatric disorders such as depression as well as in the mediation of hyperarousal seen in primary insomnia. This abnormality might represent the common risk factor, and therefore, it is quite possible that both disorders would respond to the same therapeutic intervention (eg, corticotropinreleasing hormone antagonists) [10].

\section{Methodology}

In this survey based study total number of 75 participants were involved, A questionnaire was framed in such a way that it was simple to understand and easy to answer, and questions pertaining to the general information, social history and leading questions for assessment of symptoms of sleep patterns like that of disturbed sleep and insomnia were included. Questionnaire was asked through Google forms. The questionnaire was given to participants after explaining aim of the study and instructions for filling the questionnaire and completed forms were analysed. The responses were analysed using Microsoft Excel.

\section{Results}

In the given survey, out of total participants $41 \%$ were male and 59\% were female. Around $97.3 \%$ of the participants were in the age group of 15-30 years. Upon sleep latency it was found $33 \%$ of the people need 15-30mins to get sleep after going to bed, followed by $29.3 \%$ which require less than $10 \mathrm{mins}$ to fall asleep. $44 \%$ of participants were found to have a good sleep and $41.35 \%$ experienced a sleepiness during datime.57\% people were found who had sleep for eight hours. The Figure 1.represents various reasons for waking up in midnight.

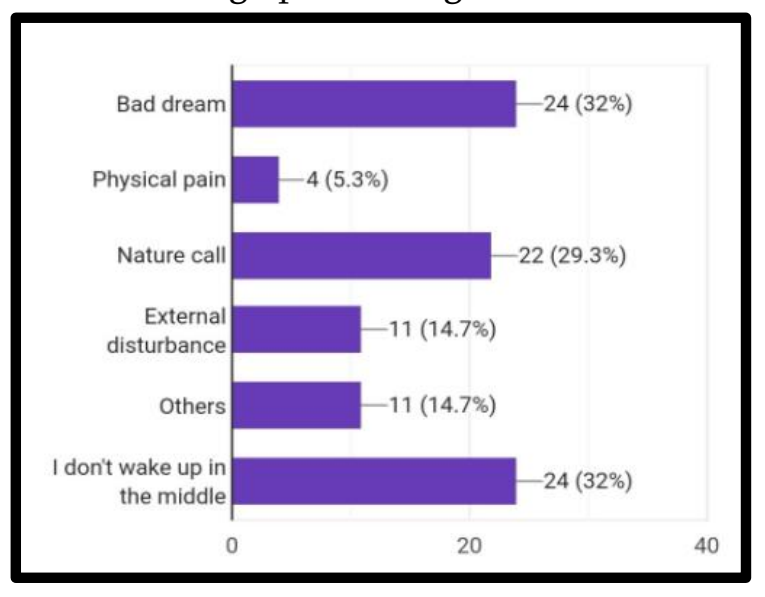

Figure 1.Graphical Representation for various reasons for disturbed sleep during midnight 
The reasons for lack of sleep were evaluated as 51\% due to work, $37 \%$ due to stress, $4 \%$ due to caffeine, $8 \%$ due to health issues.17.3\% found poor sleep impacts their professional life and $68 \%$ found that poor sleep spoils the mood for the next day. $13.35 \%$ people experienced spoiled relationship due to lack of sleep. The association between stress conditions, Quality of sleep \&Time of sleep was studied by using Pearson's correlation matrix and it showed strong correlation indicating the sleep quality of participants was affected during the COVID-19 pandemic (Table1). Hence following precautions can be taken to minimize sleep disturbance in the pandemic: Deep stretch exercises and yoga, eating early in the evening, taking a minimum of two hours break before you go to bed and avoiding the use of phone, laptops or even reading books in the bed.

Table 1. Pearson's correlation analysis

\begin{tabular}{|l|l|l|l|}
\hline & $\begin{array}{l}\text { Stress } \\
\text { Conditions }\end{array}$ & $\begin{array}{l}\text { Quality } \\
\text { of sleep }\end{array}$ & $\begin{array}{l}\text { Time of } \\
\text { Sleep }\end{array}$ \\
\hline $\begin{array}{l}\text { Stress } \\
\text { Conditions }\end{array}$ & 1 & & \\
\hline $\begin{array}{l}\text { Quality of } \\
\text { sleep }\end{array}$ & 0.629 & 1 & 1 \\
\hline Time of Sleep & 0.1 & 0.822 & 1 \\
\hline
\end{tabular}

\section{CONCLUSIONS}

During the COVID-19 lockdown, there was a worldwide increase in electronic devices' daily usage. The exposure to backlit screens before falling asleep leads to negative consequences on sleep health through its influence on the circadian system. Our investigation demonstrated a strong relationship between the modifications of the evening electronic device usage and the time course of sleep disturbances during the lockdown period. Electronic devices daily usage increased to compensate for the limited social interactions, fill free time, and ward off boredom. The increase of screen exposure in the hours before bedtime could have determined adverse consequences on sleep health. The sleep rhythms are intimately linked with the ambient light, which represents a crucial regulator of the biological clock. In light of our results, the above-mentioned interventions focused on sleep hygiene are fundamental to counteract the occurrence and exacerbation of sleep disturbances and foster the general well-being during the home confinement due to the COVID-19 pandemic.

\section{REFERENCES}

[1]. Bajaj, S., Blair, K. S., Zhang, R., Bashford-Largo, J., Dobbertin, M., \& Blair, R. J. (2020). Impact of sleep quality on the association between unease and physical exercise during initial stages of COVID-19 pandemic in India. Nature and Science of Sleep, 12, 705.

[2]. Ali, K., Mufti, U., \& Mufti, A. Impact of COVID19 Lockdown on Sleep Quality in Students: A Cross Sectional Study. Annals of International Medical and Dental Research, 6(6), 1.

[3]. Sinha, M., Pande, B., \& Sinha, R. (2020). Association of mid sleep time and social jetlag with psychosocial behaviour of Indian population during COVID-19 lockdown. Journal of Public Health Research, 9(4).

[4]. Morselli, L., Leproult, R., Balbo, M., \& Spiegel, K. (2010). Role of sleep duration in the regulation of glucose metabolism and appetite. Best practice \& research Clinical endocrinology \& metabolism, 24(5), 687-702.

[5]. Mang, G. M., \& Franken, P. (2013). Genetic dissection of sleep homeostasis. Sleep, neuronal plasticity and brain function, 25-63

[6]. Majumder, S., \& Majumder, P. (2021, September). Artificial Intelligence and Mental Health: A Study on Internet Gaming Disorder in 
COVID-19 Lockdown in India. In Interdisciplinary Research in Technology and Management: Proceedings of the International Conference on Interdisciplinary Research in Technology and Management (IRTM, 2021), 2628 February, 2021, Kolkata, India (p. 45). CRC Press.

[7]. Gupta, R., Grover, S., Basu, A., Krishnan, V., Tripathi, A., Subramanyam, A., ... \& Avasthi, A. (2020). Changes in sleep pattern and sleep quality during COVID-19 lockdown. Indian journal of psychiatry, 62(4), 370.

[8]. Basheer, R., Strecker, R. E., Thakkar, M. M., \& McCarley, R. W. (2004). Adenosine and sleepwake regulation. Progress in neurobiology, 73(6), 379-396.

[9]. Briançon-Marjollet, A., Weiszenstein, M., Henri, M., Thomas, A., Godin-Ribuot, D., \& Polak, J. (2015). The impact of sleep disorders on glucose metabolism: endocrine and molecular mechanisms. Diabetology \& metabolic syndrome, 7(1), 1-

[10]. Pace-Schott, E. F., Germain, A., \& Milad, M. R. (2015). Sleep and REM sleep disturbance in the pathophysiology of PTSD: the role of extinction memory. Biology of mood \& anxiety disorders, 5(1), 1-19.

\section{Cite this article as :}

Pragya Tiwari, Minal Trivedi, Apeksha Kesarwani, Ojaswi Gopale, "Impact of COVID-19 Lockdown on Sleep Quality in Students", International Journal of Scientific Research in Science, Engineering and Technology (IJSRSET), Online ISSN : 2394-4099, Print ISSN : 2395-1990, Volume 8 Issue 5, pp. 265-269, September-October 2021. Available at doi : https://doi.org/10.32628/IJSRSET218533 Journal URL : https://ijsrset.com/IJSRSET218533

\section{at}

